Aus dem Giesela-Kinderspital in München (Vorstand: Priv.-Doz. Dr. J. Ibrahim).

\title{
Über Tetanie der Sphinkteren, der glatten Muskeln und des Herzens bei Säuglingen.
}

\author{
Von \\ Priv.-Doz. Dr. J. Ibrahim.
}

(Mit 2 Abbildungen.)

Im Sommer des Jahres 1909 kam im Ambulatorium des GiselaKinderspitals ein Fall von Säuglingstetanie zur Beobachtung, der wegen seiner ungewöhnlichen Komplikationen der Mitteilung wert erscheint.

Fall 1. Franz B., geboren am 9. II. 1909, mit Büchsenmilch und Haferschleim ernährt, wird wegen mangelhaften Gedeihens am 1. VIII. 1909 in das Ambulatorium gebracht.

Pat. ist ein äusserst elendes Kind. Temp. 34,6, Gewicht 3370 g. Grosse Hautfalten, trockene Augen, Phimose; trinkt fast gar nichts mehr, jammert beständig. Ordination: Tee mit 0,4 Proz. NaCl und 0,4 Proz, $\mathrm{NaHCO}_{2}$.

2. VIII. Gewicht $3560 \mathrm{~g}$. Temperatur 35,6. Hat den Tee gut gegetrunken, besseres Aussehen, Teediät fortgesetzt.

3. VIII. Gewicht $3800 \mathrm{~g}$. Temp. 35,3. Ord.: $140 \mathrm{~g}$ Milch, $840 \mathrm{~g}$ Wasser, $30 \mathrm{~g}$ Nährzucker.

5. VIII. Gewicht 3710 g. Seit $1 \frac{1}{1} / 2$ Tagen kein Stuhl. Darmspülung. $5 \mathrm{~g}$ Ol. Ricin. $140 \mathrm{~g}$ Milch, $840 \mathrm{~g}$ Wasser, $20 \mathrm{~g}$ Nährzucker.

6. VIII. Pat. hat alles ausgetrunken, nicht erbrochen. - Schlaffes Aussehen, schlaffe Haltung der rechten Hand. Linke Pupille etwas grösser als die rechte. An beiden unteren Extremitäten eigenartig trockene, glänzende, etwa fingernagelgrosse rote Flecken. Ord.: $200 \mathrm{~g}$ Milch, $740 \mathrm{~g}$ Wasser, $20 \mathrm{~g}$ Kufeckemehl, $20 \mathrm{~g}$ Nährzucker.

7. VIII. 3830 g. Befinden befriedigend. Die Flecken an den Beinen sehen ganz trocken und gläuzend aus, bräunlichrot, wie eingebrannt.

9. VIII. Gewicht $3810 \mathrm{~g}$. Ordinat.: $400 \mathrm{~g}$ Milch, $540 \mathrm{~g}$ Wasser, $20 \mathrm{~g}$ Kufecke, 20 g Nährzucker.

10. VIII. Heute früh bemerkt die Mutter, dass das Kind die Beine nicht mehr bewegt; die Beine wurden immer dicker, starr und bläulich. Die Beine befinden sich in der Tat in fixierter Streckstellung, sind cyanotisch und sehr stark ödematös. Fingereindrücke hinterlassen tiefe Dellen. Das Ödem reicht bis an die Leistengegend, ist beiderseits ganz symmetrisch ausgebildet. Scrotum frei von Ödem. Die bräunlichen trockenen Hautstellen im Ödem etwas eingesenkt, aber mit der Haut emporgehoben, erheblich kleiner. Abduktion der Beine ist weniger 
behindert als die Streckung. Patellarreflexe nicht auslösbar, Plantarreflexe schwach angedeutet. - Die Füsse beide plantarflektiert, in typischer Tetaniestellung; auch die Daumen in die Hände krampfhaft eingeschlagen. - Harnblase prall gefällt, geschwulstartig durch die Bauchdecke sichtbar, erreicht Nabelhöhe. Die Phimose ist leicht zu lösen. - Durch Katheter werden $100 \mathrm{ccm} \mathrm{kl}$ aren Urins entleert. - Danach besteht die Starre weiter, hat aber schon am Ende der Blasenentleerung nachgelassen; ebenso ist die Cyanose der Beine geringer geworden.

11. VIII. Temp. 37,6. Gewicht $3900 \mathrm{~g}$. Pat. hat inzwischen einmal spontan uriniert, aber nur wenig Harn entleert. Blase wieder stark gefüllt. Ödeme und Steifheit der Beine haben erheblich abgenommen, sind aber noch deutlich vorhanden. Photographiert (s. Fig. 1. Das Ödem der Beine ist namentlich beim Vergleich mit dem abgemagerten Oberkörper zu erkennen. Am linken Unterschenkel ist noch ein Rest der Hautaffektion zu

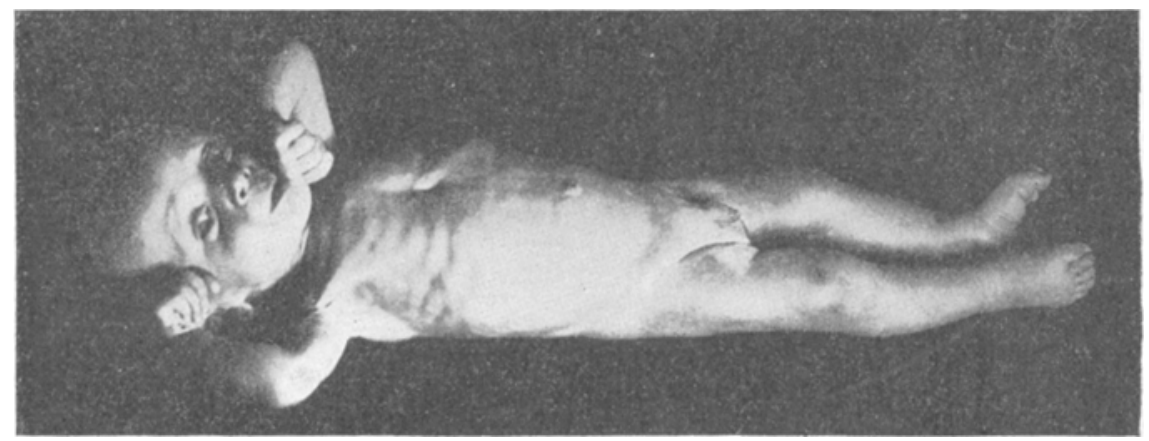

Fig. 1.

sehen). - Patellarreflexe auslösbar, nicht gesteigert. - Füsse nach wie vor in fixierter Tetaniestellung; auch die Hände in Tetaniestellungen, nicht so typisch und weniger flxiert. - Aus der Blase mit Katheter $50 \mathrm{ccm}$ leicht getrübten Urins entleert. Kein Albumen. Stuhl gelb, homogen, Jodprobe positiv. Ord.: $500 \mathrm{~g}$ Milch, $500 \mathrm{~g}$ Wasser, $20 \mathrm{~g}$ Kufecke, $20 \mathrm{~g}$ Nährzucker in $7 \mathrm{Fl}$.

12. VIII. Gewicht 3950 g. Füsse dauernd in Tetaniestellung, Hände gleichfalls. Spasmus in den Beinen hat erheblich nachgelassen. Ödeme der Beine nicht mehr nachweisbar. Leichte Ödeme an den Handrücken. Blase heute leer. Facialisphänomen nicht auslösbar. Tetaniegesicht. Galvanische Grenzwerte mit Normalelektrode am Nerv. med. in der rechten Ellenbeuge:

KSZ 1,2 ,

KÖZ wegen des KSTe nicht auslösbar,

$\mathrm{ASZ}>2,2$

$\mathrm{AOOZ}>1,4$.

13. VIII. Gewicht 3910. Tetaniestellung der Hände und Füsse konstant. Pat. sehr durstig. Stuhl homogen gelb, Jodprobe stark positiv. 
Ord.: $600 \mathrm{~g}$ Milch, $500 \mathrm{~g}$ Wasser, $20 \mathrm{~g}$ Kufecke, $20 \mathrm{~g}$ Nährzucker in 7 Flaschen.

14. VIII. Pat. sehr frisch, lacht. Immer noch ausgeprägte Tetaniestellung der Hände und Füsse. Blase leer. Facialisphänomen nicht auslösbar. Patellarreflex nicht gesteigert.

16. VIII. Gewicht $4130 \mathrm{~g}$. Fussstellung weniger krampfhaft.

19. VIII. $4180 \mathrm{~g}$.

25. VIII. Gewicht 4490 g. Fussstellung kaum mehr angedentet. Hände noch oft in habitueller, nicht fixierter Tetaniestellung. Trousseau nicht sicher auslösbar. Facialisphänomen fraglich. — Schläft gut; hat nie Glottiskrämpfe gehabt.

$$
\begin{aligned}
& \mathrm{KSZ}=1,4, \\
& \mathrm{KÖZ}=4,4, \\
& \mathrm{ASZ}=3,4, \\
& \mathrm{AÖZ}=1,8 .
\end{aligned}
$$

11. IX. Gewicht 4720 g. Kind sehr frisch. Kein Facialisphänomen. Mutter des Kindes hat Kein Facialisphänomen. Ord.: $700 \mathrm{~g}$ Milch, $400 \mathrm{~g}$ Wasser, $20 \mathrm{~g}$ Kufecke, $20 \mathrm{~g}$ Nährzucker in $6 \mathrm{Fl}$.

9. XI. Gewicht 5950 g. Krampfsymptome oder Harnverhaltung haben sich nicht mehr eingestellt. Bronchitis.

Fassen wir das Wesentlichste kurz zusammen, so ergibt sich: Ein schwer atrophisches Kind erkrankt im Alter von 6 Monaten an manifester Tetanie. Gleichzeitig stellt sich hochgradige Harnverhaltung und Ödem der Beine ein. Die Harnverhaltung schwindet nach zweimaligem Katheterismus, die Symptome der Tetanie verlieren sich allmählich im Verlauf von 14 Tagen ohne sperielle Behandlung.

Was unseren Fall auszeichnet, sind vor allem die Erscheinungen von seiten der Haut. Für die eigenartigen trockenen, erst rötlichen, später bräunlichroten glänzenden Flecken, die wie eingebrannt aussahen, kann ich eine sichere Deutung nicht geben. Ob sie als trophische Störungen aufzufassen sind? Dass es sich etwa um Verbrennungen gehandelt hätte, war mit Bestimmtheit auszuschliessen.

Die Ödeme, die bei der Säuglingstetanie häufiger vorkommen, sind meist auf Hand- und Eussrücken beschränkt, greifen höchstens noch auf das unterste Drittel des Unterschenkels über. Die ungewöhnliche Intensität der Ödeme an den Beinen in unserem Fall und deren symmetrische Ausbreitung bis herauf zur Leistengegend lassen sich vielleicht rein mechanisch als Folge von Kompression der Abdominalvenen durch die kolossal ausgedehnte Blase erklären. Mit der regelmässigen Entleerung der Blase schwanden auch die Ödeme rasch. Der Urin enthielt kein Eiweiss; eine Nephritis lag nicht vor. Die Ödeme waren auch lediglich auf die Beine beschränkt. Andererseits ist aber doch die Möglichkeit, dass es sich um angioneurotische Ödeme, also 
um vasomotorische Störungen, gehandelt haben könnte, sehr zu erwägen. Ich möchte sogar letzterer Erklärung den Vorzug geben.

Die Harn retention selbst darf wohl ganz sicher als Teilerscheinung der Tetanie betrachtet werden. Es bestand zwar eine geringe Phimose, bezw. Präputialverklebung, die aber nicht das physiologische Maß überschritt und sich leicht völlig lösen liess; es hatten sich auch nie vorher Unregelmässigkeiten in der Harnentleerung gezeigt.

Eine zweite Beobachtung von erheblicher Harnretention im Verlauf der Säuglingstetanie, die ich vor einiger Jahren als Assistent der Heidelberger Klinik machen konnte ${ }^{1}$ ), sei hier angefügt.

Fall 2. Ernst Schm. 81/2 Monate alt, wird am 10. IX. 1903 auf die Abteilung aufgenommen. Hereditär liegt nichts Besonderes vor; eine Schwester $3 \frac{1}{2}$ Jahre alt, gesund. Pat. ist rechtzeitig spontan geboren, künstlich mit Milch und Haferschleim ernährt. Seit der 6. Lebenswoche Verdauungsstörungen. Zur Zeit besteht die Nahrung aus 2 Teilen Milch und 3 Teilen Schleim. Seit 8 Tagen schwerer Durchfall, dünn, wässerig, Pat. ist nachts unruhig. In den letzten Tagen stellte sich eine ausgebreitete Furunkulose ein. 30 Furunkel wurden vom Arzt eröffnet. Pat. leidet in letzter Zeit an Stimmritzenkrämpfen, wird dabei mitunter blau.

Status: Zurückgebliebenes Kind mit sehr reduziertem Fettpolster. Gewicht 3270 g. Temperatur 37,1. Haut schlaff, faltig, nicht teigig. Am ganzen Körper verteilt zahlreiche Furunkelnarben; ein grosser Abszess am Hinterhaupt. Grosse Fontanelle weit. Schädelrachitis. Rosenkranz angedeutet. Thorax etwas fassförmig. Kein Zahn vorhanden.

Grosse Iymphdrüsenpakete am Hals, in den Inguinal-, besonders aber Axillargegenden. Pat. schnarcht laut. Kein Schnupfen. Augen und Ohren ohne Befund. Zunge feucht, belegt. Eine Aphthe an der Schleimhaut des linken Oberkiefers.

Stimme klar. Herz und Lunge zeigen normale Verhältnisse, nur l.h. u. bei tiefem Inspirium feines Knistern hörbar. - Kleiner Nabelbruch. Abdomen stark gewölbt, weich. Leber gross und ziemlich hart. Milz nicht fühlbar.

Geformter harter Kakaostuhl. Pat. trinkt gut, erbricht nicht.

Haufig leichte Glottiskrämpfe, meist im Anschluss an heftiges Schreien.

Sehr lebhaftes Facialisphänomen.

Ord.: Inzision des Abszesses. Calomel 0,01 dreistündlich, 4 Pulver im ganzen. Tee.

11. IX. Calomel 0,01 2 mal täglich. Ein schwerer Glottiskrampf.

12. IX. Ernährung mit Milchreisschleimmischungen.

15. IX. Heute 3 ganz schwere Glottiskrämpfe, zu tiefer Cyanose und allgemeiner Rigidität führend. Chloralhydrat in kleinen Dosen.

1) Herrn Prof. E. Feer bin ich für die Erlaubnis zur Publikation meiner Beobachtungen an der Heidelberger Kinderklinik zu besonderem Dank verpflichtet, dem ich auch an dieser Stelle Ausdruck geben möchte.

Deutsche Zeitschrift $\mathrm{f}$. Nervenheilkunde. $41 . \mathrm{Bd}$. 
24. IX. Trotz Chloral und Brom immer wieder leichtere oder schwerere laryngospastische Anfälle.

Heute Fieber, 40,4, ohne nachweibaren Grund. Nachts wird Pat. sehr unruhig, trinkt immer schlechter. Es stellen sich nach Nitternacht eklamptische Erscheinungen ein: Zuckungen im Nacken, in Fingern, Händen and Beinen.

25. IX. Fieber besteht fort (bis $40,8^{\circ}$ ), ist durch Wickel kaum beeinflussbar. Eklamptische Erscheinungen leichter Art sind fast beständig zu beobachten. Nur geringe Beteiligung der Glottis. Keine meningealen Symptome. Pupillen ohne Befund. Zunge wird fast beständig zum Mund herausgestreckt, ohne dass ein Grund hierfür erkennbar wäre. Kein Befund im Pharynx.

Lungen und Herz ohne jeden pathologischen Befund. Keine eigentliche Dyspnoe vorhanden. Cyanose nur im Zusammenhang mit Glottiskrämpfen.

Es bestehen offenkundige Schluckstörungen erheblicher Art, für die eine spezielle Ursache sich nicht auffinden lässt. Nasensonde stösst auf kein Hindernis. Nachmittags 2 Uhr ein schwerer Anfall von Konvulsionen mit Cyanose. Die Untersuchung des Abdomens deckt eine beträchtliche Retentio urinae auf. Obwohl die Windeln im Laufe des Tages wiederholt nass waren und auch jetzt die Windel von Urin getränkt ist, reicht die Blase bis an den Nabel. Mit dem Katheter werden $130 \mathrm{~g}$ leicht getrübten Urins entleert, der reichlich Albumen enthält. Mikroskopisch: reichlich Harnsäurekrystalle, einige Leukocyten, auch Erythrocyten, granulierte Zylinder.

Das Kind wird von der Mutter aus dem Spital nach Hause genommen. Weiterer Verlauf blieb unbekannt.

Harnretention im Verlauf der Tetanie ist auch von anderen Autoren gesehen worden. Sachs ${ }^{1}$ ) kennt das Symptom und Escherich ${ }^{2}$ ) teilt mit, dass er recht häufig, insbesondere bei Knaben, auf der Höhe des Krankheitsbildes ein auffallend seltenes Urinieren und Harnretention beobachtete. In seiner Monographie ist auch bei den kurz mitgeteilten Einzelfällen mehrfach davon die Rede; immerhin dürften so hochgradige Retentionen wie in meinen beiden Fällen ziemlich selten sein.

Der viel zitierte Fall von Oddo und Sarles ${ }^{3}$ ), der mir leider im Original nicht zugänglich war, betrifft, wie ich aus einem Referat ersehe, ein 18 Monate altes Kind. Wie bei meinem ersten Fall war die Harnverhaltung das erste Symptom, mit dem die Krankheit sich bemerkbar machte. Der Urin soll auch eiweissfrei gewesen sein; es scheint aber, dass es zu generalisierten Ödemen gekommen ist. Dio

1) B. Sachs, Lehrbuch der Nervenkrankheiten des Kindesalters. Leipzig und Wien 1897. S. 132.

2) T'h. Escherich, Die Tetanie der Kinder. Wien u. Leipzig, A. Hölder 1909. S. 63 .

3) Oddo et Sarles; Caractères des urines dans la tétanie infantile. La médecine infantile 1894. Nr. 9. 
sehr bekannt gewordene Beobachtung von Hagenbach-Burckhardt ${ }^{1}$ ) bezieht sich auf ein dreijähriges Kind. Sie sei hier kurz mitgeteilt:

Knabe. Mit 2 Jahren Konvulsionen; beständig Verdauungsstörungen. Mit 3 Jahren ist Pat. äusserst elend, zeigt hochgradige Rachitis. Blase sehr stark gefüllt: Im Urin Eiweiss. Diazoreaktion. Temp. 40,0 ${ }^{\circ}$. Konvulsionen. - Auch an den folgenden Tagen ist die Blase stets sehr gefüllt. Das Kind muss katheterisiert werden. Entleerung von $250 \mathrm{ccm}$ Harn. In den nächsten Tagen zeigen sich typische Tetaniesymptome: temporäre spastische Zustände im Facialisgebiet, Mundspitzen, starre Gesichtszüge, schmerzlich verzogene Stirn, sodann auch leichte, offenbar schmerzhafte Krämpfe beim Schlucken. Chvostek- und Trousseausches Phänomen sehr deutlich. Patellarreflexe abgeschwächt bis aufgehoben. - Der permanente Füllungszustand der Blase muss auf einen Krampfzustand des Sphinkter vesicae bezogen werden. Beim Einführen des Katheters ist stets entsprechend der prostatischen Gegend ein leichtes Hindernis zu überwinden. das oft erst nach einiger Zeit sanften Drucks nachgiebt. Blase enthält bis $300 \mathrm{ccm}$ Urin. Der Katheterismus muss während 5 Tagen ausgeführt werden. 14 Tage später waren alle Tetaniesymptome geschwunden.

Über eine Beteiligung des Sphincter ani an tetanoiden Krampfzuständen des Säuglingsalters liegt bisher keine eingehender geschilderte Beobachtung vor. Die kurzen Mitteilungen von Koeppe ${ }^{2}$ ) sollen später noch besondere Würdigung erfahren. Ich vermute, dass der Sphincter ani gar nicht so selten an den Krämpfen partizipiert. Ich habe mehrfach Fälle gesehen, in denen bei verdauungsgestörten Kindern (über deren Zugehörigkeit zur Tetanie ich allerdings nichts Generelles aussagen kann) neben eklamptischen Krampfanfällen eine intensive Gasauftreibung des Abdomens bestand; es gelang bei solchen Kindern mit "Colica flatulenta", wie dieser Zustand früher bezeichnet wurde, mitunter (nicht immer), durch Einführung eines Darmrohrs eine grosse Menge von Gasen abzulassen, und das hatte auch einen entschieden günstigen Einfluss auf das Allgemeinbefinden und auf die Krampfanfälle. Es kam auch öfters vor, dass im Verlauf des Krampfanfalls, wohl als Folge heftiger klonischer Zuckungen der Bauchmuskulatur, reichliche Flatus explosionsartig abgingen. Genauere Beobachtungen werden wohl erst mit Sicherheit die Frage entscheiden lassen, ob nicht ein Krampf des Sphincter ani in solchen Fällen zur Erklärung der klinischen Erscheinungen herangezogen werden muss.

Eine Beobachtung, die eine solche Deutung zulässt, sei etwas ausführlicher mitgeteilt! Sie stammt auch noch aus meiner Heidelberger Assistentenzeit.

1) Hagenbach-Burckhardt, Tetanie der Blase. Jahrb. f. Kinderheilk. 1899. Bd. 49. S. 111.

2) H. Koeppe, Monatsschr. f. Kinderheilkde. Bd. 6. S. 510. 
Fall 3. Peter St, wird am 1. VI. 1904 im Alter von $2 \frac{1}{2}$ Monaten auf die Säuglingsabteilung der Luisenheilanstalt anfgenommen. Eltern gesund, Pat. ist das erste Kind, rechtzeitig spontan geboren, künstlich ernährt; stets verdauungsgestört; seit 8 Tagen rein schleimige Stuhle, seit gestern auch Erbrechen. Seit 14 Tagen Fieber; kann den linken Arm nicht bewegen; seit ein paar Tagen Schwellung am linken Handgelenk bemerkt.

Status: Gewicht 4880 g. Temperatur 38,5. Kräftig entwickeltes Kind mit leidendem Gesichtsausdruck. Fahle gelbblasse Hautfarbe; etwas gedunsene Augenlider, teigige Haut am Abdomen. Grosse Fontanelle nicht eingesunken. Kein Schnupfen, keine Ohreiterung, Zunge stark weiss belegt, Rachen ohne pathologischen Befund: Skelett in Ordnung. Das linke Handgelenk geschwollen; Krepitation bei stärkeren Bewegungen; auch die linke Ellenbogengegend erscheint etwas geschwollen; der linke Arm wird geschont. - Keine Drüsenschwellungen. Innere Organe in Ordnung, $\mathbf{A b}$ domen gespannt, meteoristisch. Öfteres Erbrechen. Stuhl sehr schlecht, zerfahren; grosse Schleimmassen.

A usgesprochene Pfötchenstellung beider Hände. Bei Druck auf den rechten Oberarm (Trousseau) wird die Handstellung noch erbeblich verstärkt und fixiert. Kein Facialisphänomen, keine stärkere mechanische Erregbarkeit einzelner Nervenstämme nachweisbar. - Keine Glottiskrämpfe. Patellarreflexe beiderseits lebhaft, kein Fussklonus, Plantarreflex schwach.

Nachts 12 Uhr eklamptischer Anfall. Kurze regellose Zuckungen, besonders in den Augenmuskeln und im Facialisgebiet, aber auch in den Händen und Armen; in den Beinen treten gelegentlich auch heftigere Zuckungen auf. Glottis unbeteiligt. Auf Kneifen erfolgen Schmerzäusserungen, während die Zuckungen sich fortsetzen. Leib noch stärker aufgetrieben. Pat. krümmt sich wie in heftigen Schmerzen; es gehen mehrere Flatus ab und zugleich kleine Mengen spritzenden. Stuhls mit grossen Schleimflocken. Darmspülung entleert Gase und Schleim. Pat. schläft nach der Darmspülung ein.

Am nächsten Tag waren die Erscheinungen der manifesten Tetanie verschwunden, kebrten auch bis zur Entlassung am 1. VIII. nicht wieder. Am 2. VII. und 4. VII, wurden nochmals heftige Konvulsionen von mehrstündiger Daner beobachtet. Pat. ist dauernd aufgeregt, reizbar und zornig. Die Arthritis (Rheumatoid?) heilt völlig aus. Pat. ist später gesund geblieben, hat aber erst mit $1 \frac{3}{4}$ Jahren selbständig gehen gelernt.

Die Sphinkteren der Blase und des Darms nehmen zwischen willkürlichen und unwillkürlichen Muskeln eine Mittelstellung ein. Ihre Krämpfe beweisen nichts in der Frage, ob sich auch die glatten Muskeln an der Säuglingstetanie beteiligen können. - Auch die mehrfach erwähnten, z. B. auch in meinem zweiten und vierten Fall beobachteten Schluckstörungen, die wohl sicher als Pharynxbezw. Ösophaguskrämpfe aufgefasst werden müssen, können zur Entscheidung dieser Frage nicht herangezogen werden.

Das einzige sicher Greifbare, was hier vorliegt, ist die meines Wissens nur bei Erwachsenen nachgewiesene Beteiligung des Ci- 
liarmuskels (Kunn $\left.{ }^{1}\right)$ ) und der Irismuskulatur. Geringe Pupillendifferen $z^{2}$ ) oder Pupillenstarre ${ }^{3}$ ) finden sieh gelegentlich in der Literatur erwähnt.

In letztere Kategorie gehört zweifellos die vorübergehende $\mathrm{Pu}$ pillendifferenz in meinem 1. Falle; sie wurde leider nicht genauer studiert, so dass nicht sichersteht, ob ein Krampf des Sphinkter oder Dilatator pupillae vorlag. Einen zweiten Fall dieser Art, in dem ein Krampf des Dilatator pupillae die Grundlage der Pupillendifferenz bildete, konnte ich kürzlich beobachten. Er bietet auch sonst viel Bemerkenswertes.

Fall 4. Heinrich K., 41/2 Monate alt, wird am 13. IV. 1910 in das Gisela-Kinderspital aufgenommen. Pat. stammt von gesunden Eltern, ist das erste Kind, 6 Wochen zu früh spontan geboren, wurde 4 Monate von der Mutter gestillt, wurde abgesetzt, weil die Mutter auf Verdienst gehen musste. Von Ende März bis 10. April in einer Krippe, wurde zunächst morgens und abends noch gestillt; seit einigen Tagen in Kost, wo es Kufeckemehl und kondensierte Milch erhielt. Seit 4. IV. rapide Gewichtsabnahme.

Status am 12. IV. 1910. Gewicht $4100 \mathrm{~g}$, Länge $59 \mathrm{~cm}$, grosse Font. $2 \times 2$, Kopfumfang $37 \mathrm{~cm}$, Brustumfang $37 \mathrm{~cm}$. Sehr verfallenes Kind, blass, schlaff. Puls klein, frequent. Sofort nach der Aufnahme erfolgt heftiges Erbrechen brauner, schleimiger Massen. Magenspülung entleert viel braunen, schleimigen Mageninhalt. - Stühle massig, schaumig, dünn. - Temp. 38,7.

Kochsalzinfusion (mit Ringerscher Lösung). Kampferöl (im Laufe der Nacht 2 mal $1 \mathrm{~g}$ ) subkutan. Tee mit 0,4 Proz. Kochsalz wird gut getrunken.

13. IV. Pat. sieht heute bedeutend besser aus, ist im ganzen leidlich entwickelt. Fettpolster mässig. Kleine Nackendrüsen. Haut rein. Zunge belegt, Mundschleimhant gerötet, Lippen trocken. Sensorium völlig frei. Reflexe o. B., kein Facialisphänomen. Herz und Lange 0 . B. Leib weich, Milz nicht fühlbar. Urin frei von Eiweiss und Zucker (Redultionsproben). Sieben Stühle im Verlauf des Tages: dünne Teestühle. - Temperatur noch febril, morgens 38,0, abends 38,9. Nachts ist Pat. sehr unruhig, wälzt sich herum; warme Kataplasmen auf das Abdomen bewirken keine Besserung. Getrunkene Nahrung: $950 \mathrm{~g}$ Tee mit 0,4 Proz. Kochsalz.

14. IV. Noch sehr unruhig, fiebert noch, abends 38,8 . Aufgenommene Nahrung: $400 \mathrm{~g}$ Tee mit 0,4 Proz. Kochsalz, $400 \mathrm{~g}$ Tee mit $15 \mathrm{~g}$ Nährzucker, $80 \mathrm{~g}$ abgedrückte Frauenmilch.

15. IV. Trinkt gut; ist seit gestern Abend auffallend ruhig, beinahe

1) C. Kunn, Über Augenmuskelkrämpfe bei Tetanie. Deutsche mediz. Wochenschr. 1897. Nr. 26.

2) z. B. Feer, Korrespondenzblatt für Schweizer Ärzte. 1908. \$. 720. Escherich, Die Tetanie der Kinder. S. 69. S. 239 .

3) z. B. Finkelstein, Lehrbuch für Säuglingskrankheiten. I. 2. Teil. 
apathisch. Fontanelle nicht vorgewölbt, eher etwas eingesunken. - Ausgesprochener Dermographismus. - Pupillen und Reflexe 0. B. - Meningitis? - Nachts einmal heftig erbrochen. Stuhle sauer, dünn, 3 mal in 24 Stunden. Heute fieberfrei. Aufgenommene Nahrung: $250 \mathrm{~g}$ Tee mit Nährzucker, 250 g abgedrückte Frauenmilch.

16. IV. Pat. etwas frischer, trinkt gut. 2 Stühle etwas schleimig, alkalisch; Pat. fieberfrei; Nahrung: $350 \mathrm{~g}$ abgedrückte Frauenmilch.

17. IV. $380 \mathrm{~g}$ abgedrückte Frauenmilch.

18. IV. Versuch das Kind an die Brust zu legen, wehrt sich stark, trinkt nur wenig. Nahrung: $450 \mathrm{~g}$ Frauenmilch.

19. IV. Wird $5 \mathrm{mal}$ angelegt, trinkt besser. Nahrungsaufnahme $520 \mathrm{~g}$ Frauenmilch. - Stuhl auf Einlauf, homogen.

20. IV. Meist sehr ruhig. Auffallend langsame Bewegungen. - Präputialverklebungen gelöst. $580 \mathrm{~g}$ Frauenmilch getrunken.

21. IV. Pat. lebhafter, trinkt gut an der Brust $(590 \mathrm{~g})$. Gewicht $4080 \mathrm{~g}$. Dauernd fieberfrei.

22. IV. Die letzte Nacht war sehr unruhig. Pat. jammerte unausgesetzt, scheuerte sich Kniee und Hände wund, bohrte den Kopf in die Kissen, schwitzt stark. Nahrung (abgedrückte Frauenmilch) war nur mit dem Löffel beizubringen.

Morgens sieht Pat. sehr schlecht aus, leicht cyanotisch. Deutliche Pupillendifferenz, links weiter als rechts; beide Pupillen reagieren. Fontanelle nicht gespannt. - Ausgesprochene Nackensteifigkeit, opisthotonische Haltung. Der ganze Körper steif, gestreckt.

Atmung selır beschleunigt. Rechts vorn oben vereinzeltes Rasseln, verschärftes Atmen(?), Temperatur 37,8. Jammert fast ununterbrochen. Trinkt nicht.

Lumbalpunktion: Unter starkem Druck fliesst klare Flüssigkeit ab (15 ccm); kein vermehrter Eiweissgehalt, keine Gerinnselbildung; Reduktionsprobe positiv. Mikroskopisch: Nur sehr vereinzelte Zellen im Zentrifugat. 11/1/2 Uhr: Pat. blickt wie geistesabwesend ins Leere. Schon von weitem fällt die Pupillendifferenz auf. Rechte Pupille etwa normal, die linke erheblich erweitert. Beide reagieren (langsam) auf Lichteinfall. Atmung erheblich beschleunigt, 72, von ganz eigenartigem Typus; dauernd gleichmässiges sehr tiefes In- nnd Exspirium in Pendelrhythmus; dazwischen öfter auch Jammern und Schreien. Stimme klar; Zunge sehr trocken. Diffuse Bronchitis. Herzaktion sehr beschleunigt (140), regelmässig. Embryokardie, Beiderseits deutliches Facialisphälnomen (erstmalig konstatiert). Kopf tief in die Kissen gebohrt; exquisite Nackensteifheit. Gesicht wenig steif.

Hände in fixierter krampfhafter Fausthaltung mit maximal eingeschlagenem Daumen.

Beide Füsse in typischer Tetaniestellung, fest fixiert; leichte Ödeme auf den Fussrücken. Kniee vom Aneinanderreiben der Beine wund gescheuert; Beine im ganzen sehr steif. Abdomen gespannt, mässig aufgetrieben. Banchmuskulatur zeitweise versteift. Darmrohr schwer einführbar, da reichlich pastiger Stuhl unmittelbar über dem Sphinkter sitzt. Blase leer.

Gestern wurde $a b$ und zu tönendes Inspirium beim Schreien gehört; keine Anfälle von Glottiskrampf. Heute nichts Derartiges.

Galvanische Prüfung der Nervenerregbarkeit ist nicht möglich, da die 
Faust krampfhaft geschlossen ist und Ströme von 1,0 M.-A. schon KSTe bedingen. KSZ am rechten Medianus, soweit feststellbar, bei $0,6-0,8$ M.-A.

Deutlicher Dermographismus.

Reflexe nicht prüfbar wegen der universellen Spasmen.

An den Augen lässt sich folgender Befund erheben: Im ganzen Musc. orbicularis beiderseits besteht ein kontinuierliches feines Flimmern und Wogen, gleichsam ein feinschlägiger Tremor der aber nicht zum Schluss oder zur Verengerung der Lidspalte führt. Der Tremor erstreckt sich gleichmässig auf Ober- und Unterlid. Lidspalten nicht different.

Pupillen stark different, infolge abnormer Weite der linken Pupille. Bei seitlicher Lupenbeleuchtung verengert sich auch die linke Pupille erheblich, doch erfolgt die Reaktion langsam (wie übrigens auch rechts); die linke Pupille wird aber sofort wieder weit. Bei dauernder starker Belichtung zeigt sich ein fortwährender Wechsel zwischen Verengerung und Erweiterung.

Papille (nur links gesehen) erscheint leicht gerötet.

Um 1 Uhr ist der Atemtypus, die Herzaktion, die Pupillendifferenz unverändert; die Zuckungen im Orbicularis oculi bestehen links noch fort, haben rechts fast ganz aufgehört; um $1 \frac{1 / 2}{2}$ Uhr sind sie auch links nur noch sehr gering. Atmung immer gleich. Nackensteifheit etwas geringer. Tetanie der Hände und Füsse stets unverändert. Pat. erhält $5 \mathrm{~g}$ Ol. Ricin. und nur Tee (mit 0,25 Natr. bromat,). Da Pat, offenbar an Schluckbeschwerden leidet, erbält er um 7 Uhr den Tee mit der Schlundsonde. Etwa 10 Minuten darauf verfällt er ganz plötzlich und stirbt trotz Kampferinjektion im Zeitraum von höchstens $1 \frac{1}{2}$ Minuten. Es war ein reiner Herztod. Weder vor noch während des Todes bestand irgend ein Respirationshindernis. In- und Exspirium waren frei und hörten erst nach dem Herzstillstand auf. Die Herztöne, die unmittelbar vorher noch erheblich beschleunigt gehört worden waren, wurden successiv langsamer; sie betrugen zunächst 10 in einer Viertelminule, dann nur noch 5, zuletzt kamen nur noch einige vereinzelte Schläge, dann war es zu Ende. Es waren die beiden Herztöne bis zuletzt deutlich gesondert hörbar und rein, aber dumpf. Die Pupillendifferenz war noch nach dem Tode deutlich.

Sektion am 23. IV., vormittags $11^{1 / 2}$ Uhr (Dr. Hueck). Leiche eines etwas atrophischen Kindes mit leichter tetanischer Krampfstellung der Zehen und Finger, blassen Hautdecken und gelöster Totenstarre, sowie spärlichen Totenflecken. In der Bauchhöhle kein fremder Inhalt. Zwerchfellstand rechts 4., links 5. Rippe. Beide Pleurahöhlen leer. Im Herzbeutel die seröse Flüssigkeit ein wenig vermehrt. Schleimhant des Rachens und Ösophagus glatt und bleich. Follikelapparate des Zungengrundes gering entwickelt, ohne Einlagerungen, ebenso die trachealen Lymphdrüsen. Schilddrüse anscheinend intakt, ebenso an den beiden unteren Epithelkörperchen keine makroskopischen Veränderungen. Thymus eher etwas kleiner, von gehörigem Aussehen. Bifurkationsdrüse klein, blass.

Beide Lungen von gehöriger Grösse, mit spiegelnder Pleura überzogen, überall lufthaltig. Blutgehalt nur in den abhängigen Partien gehörig. Die vorderen eher blutarm. Saftgehalt überall reichlich. Aus den durchschnittenen Bronchien quillt überall trüber Eiter. Im linken Unterlappen etwas rahmiger Eiter. Bronchialschleimbaut dunkelrot. Auch die tracheale Schleimhaut 
mit reichlichem Schleim bedeckt. Hilusdrüsen ohne Einlagerungen, klein. Gefässe enthalten nur flüssiges Blut.

Herz klein. Beide Ventrikel fühlen sich schlaff an und enthalten beide ziemlich reichliche Cruor- und Speckgerinnsel. Herzhöhle beiderseits, besonders links, deutlich abgeflacht. Muskulatur auf Flachschnitten von blutarmer Beschaffenheit und fleckig gelbroter Farbe; überall fallen dunkler rot gefärbte, unregelmässig verwaschene Stellen auf. Klappenapparat vollständig intakt. Gefässinnenhäute glatt. Keine Gefässanomalien vorhanden.

Milz leicht vergrössert, auf dem Durchschnitt von gleichmässig dunkelroter Farbe. Pulpa nicht vorquellend. Follikel als kleinste graue Pünktchen äusserst zahlreich zu erkennen.

Leber von gehöriger Grösse, ziemlich weicher Konsistenz, auf dem Schnitt von reichlichem Blutgehalt. Azinöse Zeichnung an einigen Stellen eben sichtbar (infolge leichter Stauung). Schnittfläche sehr trübe und feucht.

Gallenblase und abführende Gallenwege intakt. Beide Nebennieren von gehöriger Grösse. Rinde intensiv gelb. Beide Nieren entsprechend gross, von glatter Oberfläche. Schnittfläche von scharfer Zeichnung, etwas feucht.

Abführende Harnwege intakt. Schleimhaut des Magendarmkanals blass, glatt. Peyersche Platten im unteren Dünndarm deutlich. Mesenteriale Lymphdrüsen ziemlich gross, blass, ohne Einlagerungen. - Pankreas ohne sichtbare Veränderungen.

Weiche und harte Schädeldecken gehörig, ebenso die Dura. Zwischen Dura und Pia die Flüssigkeit etwas vermehrt. Gefässe der Pia ziemlich stark mit Blut gefüllt. Gehirnsubstanz sehr feucht und blutreich, im ganzen von scharfer Zeichnung; nur an der Grenze zwischen linkem Stirn- und Scheitellappen ist an einigen Stellen die Grenze zwischen grauer und weisser Substanz etwas weniger scharf als sonst. Hirnventrikel nicht erweitert, Flüssigkeit ganz leicht vermehrt. Kleinhirn, Pons und Medulla von scharfer Zeichnung.

Knorpel-Knochengrenze an den Rippen nicht besonders aufgetrieben, zeigt aber auf dem Schnitt eine unscharfe Abgrenzung.

Mikroskopisch: Gehirnrinde, Herzmuskel und Parathyreoideae sind frei von irgendwelchen wesentlichen Veränderungen.

Diagno se: Tetanie. Diffuse schleimige, im linken Unterlappen eitrige Bronchitis. Dilatation besonders des linken Herzens. Geringe Rachitis. Ödem der weichen Hirnhäute.

Der geschilderte Fall verlief in mehrfacher Hinsicht ungewöhnlich. Das Kind war zur Zeit der Aufnahme ins Spital unter Teediät frei von Tetaniesymptomen. Die Tetanieerscheinungen stellten sich unter ausschliesslicher Ernährung mit Frauenmilch ein, erreichten mit dem Einsetzen einer leichten Bronchitis rapid eine ungewöhnliche Intensität und fanden mit einem akuten Herztod ihren Abschluss. Die tonischen Krampfzustände beschränkten sich nicht auf die bekannten Symptome an Händen und Füssen, sondern sie zogen auch eine Reihe anderer Muskelgebiete in Mitleidenschaft, so vor allem die Nacken-, Schlund- und Augenmuskeln. Das klinische Bild wurde dadurch dem 
einer Meningitis in so hohem Maße ähnlich, dass eigentlich nur das normale chemische und mikroskopische Verhalten der Lumbalpunktionsflüssigkeit die sichere Diagnose ermöglichte.

Von grossem Interesse war auch das Verhalten der Atmung, die nicht nur stärker beschleunigt war (72), als der Lungenbefund erwarten liess, sondern einen gerade bei der Tachypnoe ungewöhnlichen Typus darbot, ein dauernd gleichmässig vertieftes In- und Exspirium im Pendelrhythmus, wie ich es bisher bei keiner Affektion der Luftwege gesehen habe. Dabei war die Glottis, die sonst so häufig bei der Säuglingstetanie an den Krämpfen teilnimmt, fast ganz unbeteiligt. Dyspnoe und Tachypnoe als Teilerscheinungen der Säuglingstetanie sind offenbar recht selten. Escherich ${ }^{1}$ ) hat sie in einigen Fällen gesehen und Finkelstein ${ }^{2}$ ) erwähnt einen Fall von Tachypnoe (100 pro Minute) und Tachykardie (200), die unmittelbar in Anschluss an eine Magenspülung entstand und mehrere Stunden andauerte. Die mebrfach zitierte Beobachtung von Popper ${ }^{3}$ ) war der meinen offenbar nicht ganz analog, da besonders hervorgehobeu wird, dass die Atmung ungemein seicht erfolgte, ohne Zuhilfenahme der Auxiliarmuskeln. Sie betrifft auch keinen Säugling, sondern ein $3 \frac{1 / 3}{3}$ Jahre altes Kind.

Wenden wir unser Augenmerk nunmehr dem Verhalten der Augen zu. Soviel ich aus der Literatur ersehen konnte, liegen genauer geschilderte Beobachtungen über Augenmuskelkrämpfe im Verlauf der Säuglingstetanie bisher kaum vor. Es wird nur die spastische Verengerung der Augenlider bei Beteiligung der Gesichtsmuskeln an den tonischen Krampfzuständen erwähnt oder abgebildet (Wscherich4), Uffenheimer $)^{5}$ ), sowie das Vorkommen von Nystagmus und spastischem Schielen, auf das Feer ${ }^{6}$ ) speziell aufmerksam macht. Escherich ${ }^{7}$ ) erwähnt auch Krämpfe des Ciliarmuskels, die aber wohl kaum im Säuglingsalter konstatiert sein dürften. Was unseren Fall besonders auszeichnet, das ist das eigenartige Verhalten des Musculus orbi-

1) Th. Escherich, Die Tetanie der Kinder. S. 67.

2) H. Finkelstein, Lehrbuch der Säuglingskrankheiten. I. 2. Teil. S. 239. Anmerkung.

3) R. Popper, Dyspnoe bei Tetanie. Archiv für Kinderbeilkunde 1895. Bd. 18. S. 198 .

4) Th. Escherich, Die Tetanie der Kinder. S. 95.

5) A. Uffenheimer, Das Tetaniegesicht. Verhandlgn. d. Ges. f. Kinderheilkde. in Meran 1905. S. 240.

6) E. Feer, Die idiopathischen Krämpfe (Spasmophilie) des frühen Kindesalters. Korrespondenzhlatt f. Schweizer Ärzte 1908. Nr. 22. S. 714.

7) Th. Escherich. 1. c. S. 63. 
cularis, das am besten als ein feinschlägiger Tremor bezeichnet werden kann, und die spastische Pupillendifferenz, die zweifellos als Dilatatorkrampf am linken Auge aufufassen ist.

Die Tetaniesymptome treten in der Regel bilateral symmetrisch auf. Auch in unserem Fall liess die Beobachtung der Orbiculares erkennen, dass beide Augenlider am Anfalle beteiligt waren.

Dass der Dilatatorkrampf nur an einem Auge bestand, ist besonders interessant. Halbseitige Tetaniesymptome sind im grossen und ganzen recht selten; sie sind aber (bei Erwachsenen) doch in einer Reihe von Fällen beschrieben. Gerade im Bereich der Augenmuskeln scheinen sie aber häufiger vorzukommen, wie die öfter beobachtete Pupillendifferenz oder der von $\mathrm{Kunn^{1 }}{ }^{\text {) }}$ erwähnte Strabismus convergens eines Auges beweist.

Der plötzliche Herztod des Kindes, den ich zufälligerweise selbst miterlebte, soll weiter unten noch gewürdigt werden.

Die eingehendere Mitteilung des obigen Falles wurde veranlasst durch die Frage, ob sich auch die glatten Muskeln an der Säuglingstetanie beteiligen können. Für die glatte Muskulatur des Auges dürfte die Frage wohl im positiven Sinne entschieden sein. Wie steht es nun mit den anderen glatten Muskeln?

Der einzige Autor, der über Beobachtungen in dieser Richtung verfügt, ist Koeppe ${ }^{2}$ ). Er schreibt: „Sehr häufig habe ich bei Säuglingen im eklamptischen Anfall feststellen können, dass die glatte Muskulatur des unteren Rektums, ebenso wie der Sphincter externus anfallsweise sich spastisch kontrahiert. Wenn bei Tetanie nach mehreren Calomelgaben kein Stuhl erfolgte, konnte ich die Ursache davon in einem Spasmus nicht nur des Sphincter externus, sondern auch höherer Darmabschnitte finden, nach deren Lösung ausgiebiger und reichlicher Stuhl erfolgte."

Diese sehr bemerkenswerten Mitteilungen Koeppes, die allerdings nur ganz nebenher bei der Besprechung eines Falles ron Hirschsprungscher Krankheit gemacht werden und mit ausführlichen Krankengeschichten noch nicht belegt sind, haben bisher noch keine ihrer Bedeutung entsprechende Würdigung gefunden. In der grossen Escherichschen Monographie werden sie z. B. garnicht erwähnt. Ich habe zufälligerweise seit der K o eppeschen Publikation nur sehr wenige einschlägige Fälle gesehen, und es ist mir bisher nicht gelungen, analoge Beobachtungen zu machen. Diese Verhältnisse sind aber zweifellos eingehenderen Studiums wert.

1) C. $K u n n$, l. c.

2) H. Koeppe, l. c. 
Zu den glatten Muskeln, deren Krampfzustände mit der Säuglingstetanie in Beziehung gebracht werden könnten, gehört aush der Pylorus.

Es liegen aber bisher über die Beziehungen der Tetanie zu den Krankheitserscheinungen der Pylorusstenose bezw. des Pylorospasmus, jenem ziemlich scharf umschriebenen Symptomenkomplex, der sich in den ersten Lebenswochen und -monaten abspielt, nur wenig exakte Mitteilungen vor. Manifeste Tetanie wurde bei keinem dieser Kinder beobachtet. Bendix ${ }^{1}$ ) betont das Vorkommen des Facialisphänomens, das auch von Munro ${ }^{2}$ ) gesehen wurde. Meist dürfte es wohl fehlen, wie ich aus der Beobachtung einer grossen Zahl einsehlägiger Fälle weiss, bei denen ich es stets vermisste. Elektrische Grenzwerte für die galvanische Erregbarkeit sind an Säuglingen mit den Symptomen der Pylorusstenose bisher nicht mitgeteilt. Einen einzigen einschlägigen Fall konnte ich bei $\mathrm{Yanase}^{3}$ ) (Nr. 35) auffinden. Bei einem Kind, das anscheinend auf der Höhe der Krankheitserscheinungen der Pylorusstenose an akuter Herzsehwäche im Alter von 26 Tagen starb, war an einzelnen Tagen gelegentich die $\mathrm{KÖZ}<5 \mathrm{M}$.-A., in der Regel war sie aber $>5$ M.-A., speziell in den ersten Tagen nach der Aufnahme ins Spital, zu einer Zeit also, wo die spastischen Erscheinungen am Pylorus jedenfalls auf voller Höhe waren. Man wird kaum geneigt sein, aus diesen Befunden einen Zusammenhang zwischen Pylorusstenose und Säuglingstetanie abzuleiten.

Ich selbst war dieser Tage in der Lage, bei einem schweren Fall von Pylorusstenose auf der Höhe des Krankheitsbildes die elektrischen Grenzwerte am rechten Medianus zu erheben. Das Kind war am Tage der Untersuchung 10 Wochen alt. Die Werte betrugen KSZ $=4$ M.-A. - KÖZ $>6$ M.-A. - $\mathrm{ASZ}=5$ M.-A. - $\mathrm{AÖZ}>6$ M.-A.

Krampfanfälle sind bei Säuglingen mit Pylorusstenose allerdings öfters gesehen worden (in etwa 3 Proz. der Fälle). Wir wissen von den meisten Krampfanfällen in den ersten drei Lebensmonaten bisher überhaupt nicht, ob sie mit der Tetanie in Zusammenhang gebracht werden können, für die terminalen Konvulsionen, wie sie bei diesen Kindern beobachtet wurden, ist das zum mindesten äusserst unwahrseheinlich.

Während ich also glaube, dass die Tetanie für die Krankheitsfälle, die in der pädiatrischen Literatur als Pylorospasmus oder (angeborene)

1) B. Bendix, Die chronischen Verdauungsstörungen des Säuglingsalters in: Die deutsche Klinik. Bd. 7. 1905. S. 630 .

2) J. C. Munro, Operations of the stomach with report of cases. Boston med. and surg. Journ. 1904. II, S. 189.

3) J. Yauase, Über Epithelkörperbefunde bei galvanischer Übererregbarkeit der Kinder. - Jahrb. f. Kinderheilkde. 1908. Bd. 67. S. 98. 
Pylorusstenose bezeichnet werden, keine pathogenetische Bedeutung hat, scheint mir andererseits die Frage doch sehr diskutabel, ob nicht im Verlauf der Säuglingstetanie Pylorospasmen vorkommen.

Spezielle Untersuchungen über die Magenentleerung bei der Säuglingstetanie, über event. Ischochymie, Hyperacidität, Magenperistaltik usw. liegen nicht vor.

Wie sich aber ein Pylorospasmus im späteren Säuglingsalter klinisch charakterisiert, daräber wissen wir bisher nur sehr wenig. Es wäre wohl denkbar, dass sich die Erscheinungen erheblich weniger stürmisch geltend machen als in den ersten Lebenswochen, in denen das explosive Erbrechen und die hyperkinetischen Symptome der Magenwand das Krankheitsbild beherrschen. Es wäre daher auch wohl möglich, dass sich durch zielbewusste Untersuchungen gelegentlich Pyloruskrämpfe bei tetaniekranken Säuglingen nachweisen lassen.

Zu den glatten Muskeln, die sich an den Tetaniekrämpfen beteiligen könnten, gehört ferner die Gefässmuskulatur. Angiospastische Erscheinungen bei erwachsenen Tetanikern hat H. Curschmann ${ }^{1}$ ) erst neuerdings beschrieben. Beim Säugling sind solche Gefässkrämpfe bisher noch nicht beobachtet worden. Dass aber vasomotorische Störungen vorkommen, ist bekannt, und als solche dürften wohl anch die symmetrischen $0 ̈$ deme der Beine bei meinem Fall 1 aufgefasst werden. Auch die bei Säuglingen so häufig als Begleiterscheinung der Karpopedalspasmen sich einstellenden Ödeme an Hand- und Fussrücken sind wahrscheinlich analog zu erklären.

Der Vollständigkeit halber seien noch die Bronchialmuskeln erwähnt, sowie die glatten Muskeln im Bereich des Genitalapparats, welch letatere bei krampfhafter Kontraktion z. B. eine Erektion bewirken könnten. Ich bin nicht in der Lage, irgend welche Beobachtungen hierüber namhaft zu machen.

Zum Schluss möchte ich noch die Frage erörtern, ob nicht auch der Herzmuskel:von der Tetanie ergriffen werden kann. Das Einzige, was ich hierüber in der Literatur auffinden konnte, ist eine Bemerkung von Escherich 2), der eine Beteiligung des Herzmuskels in Abrede stellt, und die bereits erwähnte kurze Notiz von Finkelstein ${ }^{3}$ ) über einen Fall, bei dem unmittelbar im Anschluss an eine Magen-

I) H. Curschmann, Über einige ungewöhnliche Ursachen und Syndrome der Tetanie bei Erwachsenen nebst Vorschlägen zu ihrer Behandlung. - Deutsche Zeitschr. f. Nervenheilkde. 1910. Bd. 39. S. $36 \mathrm{ff}$.

2) Th. Escherich, l. c. S. 63.

3) H. Finkelstein, Lehrbuch der Säuglingskrankheiten. I. 2. Teil. S. 239, Anmerkung. 
spülung Tachypnoe (100 pro Minute) und Tachykardie (200) entstand und mebrere Stunden andauerte.

Ich habe mir eine von der allgemein gültigen Ansicht völlig abweichende Vorstellung gebildet und möchte sie im Folgenden, näher begründen.

Es ist eine Tatsache, über die alle Autoren einig sind, dass im Verlauf der Säuglingstetanie gar nicht selten plötzliche unerwartete Todesfälle vorkommen. Diese plötzlichen Todesfälle erfolgen zum Teil im Anschluss an einen Stimmritzenkrampf, gelegentlich aber auch, ohne dass ein solcher nachgewiesen werden könnte. Man ist dann genötigt anzunehmen, dass ein unbeobachteter Anfall von exspiratorischer Apnoe (Kassowitz $\left.{ }^{1}\right)$ ) dem Leben ein Ziel setzte.

Es ist aber eine allen klinischen Beobachtern seit Pott ${ }^{2}$ ) und Escherich ${ }^{3}$ ) geläufige Tatsache, die ich auch selbst wiederholt bestätigen konnte, dass der Tod dieser Kinder im Stimmritzenkrampf bezw. im Anschluss an die exspiratorische Apnoe kein Erstickungs-, sondern ein Herztod ist. Die künstliche Atmung, die Intubation, Tracheotomie usw. kommen fast immer zu spät, auch wenn der Arzt selbst bei dem Anfall zugegen ist; es gelingt nicht mebr, die Kinder zum Leben zu erwecken, was doch in wirklichen Erstickungsfällen, z. B. bei der Kehlkopfdiphtherie in einem grossen Teil der Fälle möglich ist.

Escherich ${ }^{4}$ spricht sich dahin aus, dass es sich „um ein Versagen des Atemzentrums, in den meisten Fällen aber um einen gleichzeitigen Stillstand des Herzens, eine Synkope handelt, für welche da, wo er vorhanden, der bestehende Status lymphaticus herangezogen werden könnte. Sicher sei, dass die sogenannten plötzlichen Todesfälle bei Tetanie fast ausschliesslich pastöse Kinder mit grosser Thymus betreffen".

Nun gibt es aber genug Fälle, wo der Status lymphaticus und die grosse Thymus bei der Sektion vermisst werden. Ganghofner ${ }^{5}$ ) teilt z. B. in seinem bekannten Referat äber plötzliche Todesfälle im Kindesalter mit, dass er im Laufe von 7 Jahren 8 Fälle von plötz-

1) Kassowitz, Verhandlungen der Gesellschaft für Kinderheilkunde in Karlsbad 1902. S. 81.

2) Pott, Über Thymusdrüsenhyperplasie und die dadurch bedingte Lebensgefahr. Jahrb. f. Kinderhlkde. 1892. Bd. 34. S. 130.

3) Th. Escherich, Bemerkungen über den Status lymphaticus der Kinder. Berl. klin. Wochenschr. 1896. Nr. 29.

4) Th. Escherich, Die Tetanie der Kinder. S. 66.

5) Ganghofner, Plötzliche Todesfälle im Kindesalter. Verhandlungen der Gesellschaft f. Kinderheilkunde in Kassel 1902. S. 58. 
lichem Tod bei Laryngospasmus erlebt und obduziert habe. Unter diesen 8 Fällen zeigten 6 keine Vergrösserung der Thymus und nur bei 2 erschien sie etwas grösser, doch überschritt ihr Gewicht auch bei diesen 2 nicht die Durchschnittszahlen der Norm. Eine deutliche Hyperplasie des übrigen lymphatischen Apparats war in 5 Fällen vorhanden, die anderen 3 boten nichts vom Status lymphaticus. - Auch unter den plötzlichen Todesfällen, die Pott ${ }^{1}$ ) so anschaulich beschreibt, befindet sich einer (Fall 1), bei dem die Thymusdrise ein normales Durchschnittsgewicht aufwies, und es liesse sich sicherlich leicht noch eine grössere Zahl analoger Fälle sammeln.

Wie ist nun aber bei diesen Kindern der plötzliche Herzstillstand zu erklären, namentlich in den Fällen, in denen laryngospastische Anfälle überhaupt nicht beobachtet wurden?

Ich glaube, dass wenigstens in einem Teil dieser Fälle das plötzliche Versagen des Herzens als primäres Tetaniesymptom zu deuten ist, als eine den Karpopedalspasmen, dem Glottiskrampf, der Eklampsie koordinierte Erscheinung; ich möchte vorschlagen, es als Herztetanie zu bezeichnen.

Diese Auffassung macht für die meisten Fälle alle mehr oder weniger gekünstelten Erklärungen entbehrlich, die sich auf den Status thymicus oder Status lymphaticus beziehen. Diese Erklärungen versagen überall da, wo die Obduktion einen ausgeprägten Status lymphaticus vermissen lässt. Ausserdem ist aber mit der Geltendmachung des Status lymphaticus der plötzliche Tod auch in seiner Pathogenese keineswegs klar gestellt. Denn warum beim Lymphatiker das Herz mitunter plötzlich versagt, das weiss man bisher gerade so wenig wie beim Tetaniker.

Wenn aber feststeht, dass die gesamte quergestreifte Muskulatur (einschliesslich der Sphinkteren) und sogar ein Teil der glatten Muskulatur (Ciliarmuskel, Iris, Gefässmuskeln) von tetanoiden Krämpfen befallen werden können, scheint mir die Möglichkeit, dass auch der (quergestreifte) Herzmuskel mit in Leidenschaft gezogen werden kann, durchaus nicht fern zu liegen; die Nerven, denen ein Einfluss auf die Herzbewegung zukommt, sind der Vagus und der Sympathicus. Wir wissen, dass Muskeln aus dem Innervationsgebiet beider Nerven sich an tetanoiden Krampfzuständen beteiligen können; rom Vagus ist das ja längst bekannt (Glottiskrämpfe, Schlingkrämpfe); für den Sympathicus dürfte die oben mitgeteilte Beobachtung (Dilatator pupillae) den Beweis erbringen.

Das rhythmisch arbeitende Herz kann ja nun mit der willkür-

1) Pott, l. c. S. 120 und 121. 
lichen Extremitätenmuskulatur nicht ohne weiteres verglichen werden. Versuchen wir aber einmal, uns vorzustellen, welche Wirkung ein Reiz ausüben müsste, der in den Bahnen der Herznerven verliefe und ana$\log$ wäre den Reizen, die an den Extremitäten die bekannten Dauerspasmen bewirken. Ein derartiger Krampf am Herzen könnte sich nur durch die Unterbrechung der rhythmischen Tätigkeit, durch den Stillstand des Herzens äussern. Damit soll keineswegs gesagt sein, dass etwa ein tonischer Krampfzustand der Muskulatur des Herzens oder einzelner Herzabschnitte, also ein Herztetanus, zustande kommen müsste. $\nabla$ on der experimentellen Vagusreizung wissen wir ja, dass sie einen Stillstand des Herzens in Diastole zur Folge hat. Es wäre denkbar, dass der Herztod im Verlauf der Säuglingstetanie auf diese Weise zustande käme; bei der Mannigfaltigkeit der nervösen Einflüsse, von denen die Herztätigkeit beherrscht wird, wäre es aber auch sehr wohl möglich, dass nicht jeder Fall in gleicher Weise zu deuten wäre.

Ich hatte in den letzten Monaten Gelegenheit, drei Fälle von plötzlichem Herztod im Verlauf der Sänglingstetanie zu beobachten, Der eine Fall ist bereits oben eingehend geschildert. Die beiden anderen seien zunächst in Kürze mitgeteilt:

Fall 5. Therese S. wird im Alter von $91_{4}^{1 / 4}$ Monaten am 6. III. 1910 in das Gisela-Kinderspital aufgenommen. Eltern sind gesund. Bei der Mutter lässt sich ein deutliches Facialisphänomen auslösen. Das erste Kind ist 6 Jahre alt, gesund, das zweite ist 3 Jahre alt, leidet an Rachitis, das dritte Kind litt an Krämpfen. - Pat. ist das vierte Kind, ist rechtzeitig spontan geboren, künstlich ernährt mit Kuhmilch und Reisschleim, nie recht gediehen. - Seit einiger Zeit zieht es beim Schreien, seit gestern schmerzhafte Krampfhaltung der Hände und Füsse; keine eklamptischen Erscheinungen.

Status: Gewicht $4930 \mathrm{~g}$, Länge $60 \mathrm{~cm}$, Temp. 37,2 .

Unterernährtes Kind mit ausgeprägten Zeichen der Rachitis (grosse Fontanelle, weiche Nähte, Rosenkranz). Kein ausgeprägtes Tetaniegesicht.

Beide Hände und Füsse in typischer, maximal fixierter Tetaniestellung, die gar nicht ausgeglichen werden kann. Auch die Muskeln der Arme und Beine rigid. Facialisphänomen stark vorhanden. Keine Laryngospasmen beim Schreien, keine klonischen Krämpfe.

Thymusdämpfung den Sternalrand etwas überragend.

Innere Organe ohne pathologischen Befund. Ord.: $10 \mathrm{~g}$ Ol. Ricin. Schleimdiät. Protrahiertes warmes Bad.

Die Krämpfe lösen sich auch im Bad nicht. Nahrungsaufnahme gut. Das Kind ist munter, weint aber, sowie man es anrühren will. Um 9 Uhr abends wird Pat, tot im Bett gefunden. Die Schwester hatte es noch 10 Minuten zuvor besichtigt und keine Veränderung in seinem Befinden bemerkt; sie hatte den Saal nicht verlassen. Der Tod war obne hörbaren Todeskampf erfolgt. Der sofort herbeigerufene Arzt konstatierte eine leichte Cyanose der Nägel und Lippen; die Füsse und Hände befanden sich nach wie vor in der typischen Kontrakturstellung, wie sie die beige- 
gebene, am nächsten Morgen aufgenommene Photographie erkennen lässt. Der Kehlkopf war geöffnet, Intubation ohne jede Schwierigkeit ausfúhrbar. Künstliche Atmung blieb erfolglos.

Sektionsbefund am 10. III. morgens $11 / / 2$ Uhr $\left(14 \frac{1}{2}\right.$ h. p. mort.). Etwas zurückgebliebenes Kind in mässigem Ernährungszustand. Hände and Füsse in fixierter Tetaniestellung. Situs der Eingeweide normal; kein abnormer Hochstand des Zwerchfells. Coecum ganz frei beweglich; langer Wurmfortsatz. Pylorus und Antrum pyloricum fest kontrahiert, aber nicht abnorm hart anzufühlen; die Därme zum grössten Teil erschlafft; einige Dündarmschlingen mässig kontrahiert. Mesenterialdrusen durchweg erheblich vergrössert. - Leber von normaler Farbe und Konsistenz. - Milz etwas vergrössert, $24,5 \mathrm{~g}$, ziemlich hart, Follikel sehr deutlich weissgrau. - Nieren hyperämisch, nicht getrübt, Nebennieren ziemlich klein, besonders die Marksubstanz schmal. Gewicht beider Nebennieren zusammen $3 \mathrm{~g}$. Magen fast leer. Pylorus zeigt auf dem Durchschnitt normale Verhältnisse.

Th y m u s mässig gross, $14,8 \mathrm{~g}$, reicht bis zur Mitte des Herzbeutels.

Lungen blutreich, Iufthaltig, ohne pathologischen Befund. Pleura nirgends adhärent, durchsichtig, nur an einer kleinen Stelle am vorderen Rand des rechten Oberund Unterlappens einige kleine subpleurale Effusionen. Trachea und Ösophagus ohne Befund. Keine Hypertrophie der lymphatischen Gebilde des Rachenrings.

Fig. 2.

Herz 32,5 g. Rechter Ventrikel völlig erschlafft, linker Ventrikel ungewöhnlich fest kontrahiert and hart. Herz von normaler Grösse. Klappen ohne Befund. Herzfleisch makroskopisch nicht verändert.

Rippen-Rippenknorpelgrenze zeigt auf dem Durchschnitt erhebliche rachitische Veränderungen.

Gehirnsektion musste aus äusseren Gründen unterbleiben.

Von einem sehr ausgeprägten Status thymico-lymphaticus kann im vorliegenden Fall wohl kaum die Rede sein; die Mesenterialdrüsen- 
schwellung kann ihre Erklärung sehr wohl in den chronischen Verdauungsstörungen finden, an denen das Kind seit seiner Geburt zu leiden hatte; die vergrösserte Milz kann mit der Rachitis zusammenhängen; die Thymus wiegt nach den bekannten Erhebungen von Fried leben ${ }^{1)}$ bei der Geburt durchschnittlich 14,3 g, vom 1.-9. Monat 20,7 g, rom 9.-14. Monat 27,3 g. v. Sury ${ }^{2}$ ) gibt als Durchschnittsgewicht für das Alter vom 2.-9. Monat $16 \mathrm{~g}$ an. Thr Gewicht hielt sich also bei dem über 9 Monate alten Kinde mit 14,8 $\mathrm{g}$ unter dem Durchschnittsgewicht. Immerhin ist zuzugeben, dass sie verhältnismässig gross war, wenn man den reduzierten Ernährungszustand des Kindes berücksichtigt. Wir wissen ja, dass die Thymus bei atrophischen Säuglingen meist erheblich an Gewicht einbüsst. - Ein mässiger Grad von Status lymphaticus dürfte also wohl vorgelegen haben.

Fall 6. Karl M. wird am 17. IV. 1910 im Alter von 10 Monaten ins Gisela-Kinderspital, aufgenommen. Pat. ist ein illegit. Kind. Mutter leidet an Rheumatismus. Pat. ist das erste Kind, rechtzeitig spontan geboren. 14 Tage gestillt, dann mit Milch und Gerstenschleim ernährt. Seit 3 Monaten Vollmilch und Semmelmus. Mehrfach Verdauungsstörungen. Seit 3 Tage zieht es beim Schreien auf. Heute früh ein kurzdauernder Anfall von Atemnot mit Blauwerden und allgemeiner Steifheit des Körpers. Solche Anfälle wiederholten sich noch 2 mal.

Sta tu s bei der Aufnahme: Mässiger Ernährungszustand. Gewicht $5520 \mathrm{~g}$. Länge $63 \mathrm{~cm}$. Afebrile Temperatur. Grosse Fontanelle weit offen. Thoraxrachitis. Keine Epiphysenauftreibung an den Extremitäten. Sitzt noch nicht. Kein Zahn.

Innere Organe zeigen normalen Befund. Milz nicht fühlbar.

Pat. ist aufgeregt, aber freundlich. Leichtes Ziehen beim Schreien. Kein ausgeprägtes Tetaniegesicht. Facialisphänomen auslösbar; ebenso das Trousse ausche Zeichen.

Galvanische Schwellenwerte mit Stintzingscher Normalelektrode am rechten Medianus in der Ellenbeuge:

$$
\begin{aligned}
\mathrm{KSZ} & =0,8 \mathrm{M} .-\mathrm{A} . \\
\mathrm{KOOZ} & =2,2-2,4 \mathrm{M} .-\mathrm{A} . \\
\mathrm{ASZ} & =1,6-1,8 \mathrm{M} .-\mathrm{A} . \\
\mathrm{AÖZ} & =1,2 \mathrm{M} .-\mathrm{A} .
\end{aligned}
$$

18. IV. Nachts ein Anfall von ca. 4 Minuten Dauer. Kind wird blau und steif; keine Zuckungen, kein Krähen. KÖZ $=2,6 \mathrm{M}$.-A. - Nachmittag Krampfanfall von ca. 1 Minute Dauer, wird beim Fütterungsversuch blau und steif, verdreht die Augen. Keine Zuckungen. 0,5 Chloralhydrat.

Ernährung mit Finkelstein-Suppe. Im Laufe der nächsten 14 Tage erfolgten keine Anfälle mehr, nur gelegentlich war beim Schreien ein

1) A. Friedleben, Die Physiologie der Thymusdrüse usw. Frankfurt 1858.

2) K. v. Sury, Über die fraglichen Beziehungen der sogenannten Mors thymica zu den plötzlichen Todesfällen im Kindesalter. - Vierteljahrsschrift für gerichtliche Medizin und öffentliches Sanitätswesen. 3. Folge. Bd. 36. H. 1. 
leicht krähendes Inspirium zu hören. Die Behandlung bestand in Ernährung mit F inkelste in-Suppe. Eine Woche lang wurde Calcium lacticum (3 g pro die) gegeben. Das Körpergewicht hielt sich etwa auf gleicher Höhe wie bei der Aufnahme. Temperaturen waren normal. Bei der elektrischen Untersuchung bestand dauernde Übererregbarkeit (tägliche Untersuchung). Die KÖZ schwankte zwischen 1,6 M.-A. und 4,4 M.-A.

Am 2. V. wird wieder leichtes Ziehen beim Schreien bemerkt. Nachts ist Pat. unruhig gewesen, hat viel geschrieen, scheint sich in Bauch-Seitenlage zu beruhigen.

Um 10 Uhr a. m. erhält er $25 \mathrm{ccm} 3$ proz. Natr. bromat.-Lösung $=0,75 \mathrm{NaBr}$.

Um 11 Uhr a. m. die gleiche Dosis.

Pat. schläft danach nicht, ist munter, lacht viel; $1 \frac{1 / 2}{1}$ Uhr p. m. einmal Ziehen.

2 Uhr p. m. KÖZ $=2,6 \mathrm{M}$-A., KSZ $=1,6 \mathrm{M}$-A. Facialisphänomen deutlich, schreit vor der Flasche um $6 \mathrm{Uhr}$ viel, beruhigt sich aber sofort beim Trinken und ist danach sehr vergnügt. $6 \frac{1}{2}$ Uhr p. $\mathrm{m}$. KÖZ $=2,2 \mathrm{M}$.-A.; $\mathrm{KSZ}=1,0 \mathrm{M}$.-A., erhält um $63 / 4$ Uhr und $73 / 4$ Uhr je $0,75 \mathrm{~g}$ Natr. bromat. Um 1/2 8 Uhr schreit Pat. viel, ohne zu ziehen, beruhigt sich beim Herausnehmen, schreit aber bald wieder, da er hingelegt wird. Wird auf den Bauch gelegt. Vom Nebenzimmer aus hört die Schwester ihn noch ca. 15 Minuten lang schreien (ohne krähende Inspiration); dann wird er still. Als die Schwester 5 Minuten danach an sein Bett kommt, ist er tot $(1 / 29 \mathrm{Uhr})$. Der herbeigerufene Arzt konstatiert: krein Puls, keine Herztöne, keine Cyanose.

Auch die Obduktion (Dr. Hueck) ergibt keinen Anhaltspunkt für einen Erstickungstod, der etwa durch die Bauchlage hätte verschuldet sein können.

Sektion am 3.V.1910 um 12 Uhr mittags (151/2 Stunden post mortem). Hautdecken blass, Totenstarre fast nicht vorhanden. Totenflecke spärlich. Fettpolster und Muskulatur gut entwickelt. In der Bauchhöhle die Organe in gehöriger Lagerung. Flüssigkeit leicht vermehrt. Zwerchfellstand beiderseits 4. Rippe. Im Herzbeutel ebenfalls etwas vermehrte Flüssigkeit. Beide Lungen frei. Pleurahöhlen leer.

Schleimhaut der Halsorgane und der Speiseröhre mässig rot, glatt. Follikelapparate im Zungengrund und beide Tonsillen stark ausgebildet, enthalten keine Einlagerungen. Tracheale Lymphdrüsen gross, etwas feucht, ohne sichtbare Veränderungen, ebenso Schilddrüse und Nebenschilddrüsen intakt.

Thymus nicht vergrössert, wiegt $8 \mathrm{~g}$. Schnittfläche zeigt die gewöhnliche grauweisse Beschaffenheit.

Herz von entsprechender Grösse. Linker Ventrikel fühlt sich fest an, rechter ganz schlaff. Beim Aufschneiden erweist sich letzterer als stark erweitert, enthält fast nur flüssiges Blut und ganz spärliche Gerinnsel. Auch der linke Ventrikel von etwas rundlicher Gestalt. Trabekeln abgeplattet. Wand von gehöriger Dicke. Herzmuskulatur auf Flachschnitten blass graurot, ohne Einlagerungen. Klappenapparate und Innenhäute der grossen Gefässe vollständig glatt und intakt. 
Beide Lungen von gehöriger Grösse, überall weich und lufthaltig. Schnittfläche allenthalben glatt. Saft- und Blutgehalt reichlich, nirgends Einlagerungen. Bronchien, Hilusdrüsen und Gefässe ohne Veränderungen.

Milz ganz wenig vergrössert, fest; auf der Schnittfäche die Follikel als ungewöhnlich grosse, grauweisse Körner sichtbar. Pulpa dunkelrot, nicht abstreifbar.

Leber etwas gross, fest, sehr blut- und saftreich, von ganz verwaschener Zeichnung. Gallenblase und Gallenwege intakt.

Beide Nebennieren blass, Rinde gelb, Mark sehr spärlich. Beide Nieren von gehöriger Grösse, blutreich, feucht, von sehr scharfer Zeichnung. Nierenbecken, Harnleiter und Blase intakt.

Magendarmkanal zeigt völlig glatte, grauweisse Schleimhaut. Lymphapparate kräftig, ebenso Lymphdrüsen des Mesenterium. Darminhalt gehörig.

Weiche und knöcherne Schädeldecken ohne Befund. Dura und Pia vollkommen glatt. Hirnsubstanz auf der Schnittfäche sehr feucht, ziemlich blutreich. Zeichnung in allen Teilen durchaus scharf.

Knorpelknochengrenze an den Rippen verwaschen. Marksubstanz in feinen Linien in die Knorpelmassen hineinwuchernd.

Diagnose: Dilatation besonders des rechten Herzens. Akute Stauung und Ödem der Parenclyyme. Mässiger Status lymphaticus. Rachitis.

Überblicken wir die drei Fälle, so zeigen sie untereinander ausser dem plötzlichen Herztod wenig Gemeinsames. Dass es sich bei keinem der drei Kinder um einen Erstickungstod oder einen Tod im Glottiskrampf handelte, halten wir für sichergestellt. Im einen Fall (Nr.4) ergiebt sich das aus der unmittelbaren ärztlichen Beobachtung, im Fall 5 hatten Glottiskrämpfe überhaupt keine Rolle gespielt. Es wäre wohl eine rein willkürliche Annahme, dass hier eine exspiratorische Apnoe den Tod bedingt hätte. Die Sektion ergab nichts, was auf einen Erstickungstod hätte schliessen lassen. Das Gleiche gilt für den letzten Fall (6), in dem zu Beginn des Leidens allerdings Atemstörungen mit Beteiligung der Stimmbänder zur Beobachtung kamen. Im weiteren Verlauf der Krankheit waren diese Atemkrämpfe völlig weggeblieben und das Fehlen jeglichen Erstickungszeichens bei der Sektion lässt auch hier meines Erachtens sicher einen solchen Anfall als Todesursache ausschliessen; es lässt sich aus gleichem Grund auch mit Bestimmtheit behaupten, dass nicht etwa das in Bauchlage befindliche Kind durch einen dieser Lage zuzuschreibenden unglücklichen Zufall erstickt ist.

Klinisch boten die drei Fälle drei verschiedene Typen der Säuglingstetanie; der erste Fall, in vielfacher Hinsicht ganz ungewöhnlich, namentlich durch sein akutes Entstehen bei ausschliesslicher Ernährung mit Frauenmilch und durch den rapiden tödlichen Verlauf, gehört jeden- 
falls zu den schwersten Fällen von Säuglingstetanie überhaupt. Bei ihm spielten Atemstörungen im Krankheitsbild wohl eine Rolle, doch wurden Glottiskrämpfe nicht beobachtet. Von einem Status thymicolymphaticus wurde bei der Sektion nichts gefunden.

Die beiden anderen Fälle zeigten leichte Grade von Status lymphaticus; bei einem war auch die Thymus etwas grösser, als man sie im Verhältnis zum geringen Ernährungszustand des Kindes erwartet hätte; doch war von einer Hypertrophie der Thymus keine Rede und auch die sonstigen Anomalien bewegten sich in so mässigen Grenzen, dass es nicht berechtigt sein dürfte, sie direkt als Ursache für den plötzlichen Tod anzuschuldigen. Solchen leichten Graden von Lymphatismus begegnet man erfahrungsgemäss sehr häufig; in ihrem klinischen Verlauf war die Tetanie bei diesen beiden Kindern ganz different aufgetreten. Der eine bot das bei Säuglingen im grossen und ganzen nicht häufige Bild der persistenten manifesten Tetanie. Eklamptische Anfälle hatte er nie gehabt, auch keine Glottiskrämpfe irgend erheblicher Art. Während des kurzen Spitalaufenthaltes war ein tönendes Inspirium überhaupt nicht beobachtet worden.

Der letzte Fall dagegen war einer jener häufigsten Typen von Säuglingstetanie, bei dem keine manifesten Krämpfe der Extremitätenmuskulatur zur Beobachtung kamen, dagegen anfallsweise spastischer Stimmritzenverschluss mit A temstillstand und Übergreifen der Krämpfe auf die Körpermuskulatur; beim Schreien war ein tönendes Inspirium auch in den anfallsfreien Zeiten häufig zu hören.

Alles in allem können wir sagen, dass die drei klinischen Bilder völlig different waren, so dass man auf Grund meiner Fälle kaum in der Lage ist, aus dem klinischen Verlauf einen Anhaltspunkt zu gewinnen, ob ein Kind von der Herztetanie bedroht ist oder nicht. Dass ein Status thymico-lymphaticus ein besonders disponierendes Moment ist, ergiebt sich nicht gerade aus den obigen Fällen, ist aber eine schon längst gesicherte Tatsache.

Wenden wir unser Augenmerk nunmehr den Sektionsbefunden am Herzen zu! In zwei Fällen (Nr. 4 u. 6) war das Herz etwas dilatiert und schlaff, der Befund also mit einer im Vagus verlaufenden Reizung vereinbar; im dritten Fall (Nr. 5) war das rechte Herz schlaff, der linke Ventrikel jedoch ungewöhnlich fest kontrahiert und hart. Man könnte daran denken, dass gelegentlich der Anfall von Herztetanie selbst überwunden sein könnte, das Kind aber doch an den Folgen des Anfalls zugrunde ginge und auf diese Weise ein einheitlicher Sektionsbefund am Herzen gar nicht zu erwarten wäre. Mir scheint es aber viel wahrscheinlicher, wie schon oben angedeutet wurde, dass nicht nur die im Vagus verlaufenden Herznerven von der Tetanie 
beeinflusst werden können, sondern ebenso gut auch gelegentlich die vom Sympathicus stammenden Nerven, so dass nicht alle Fälle nach einem Gesichtspunkte zu beurteilen wären. Wir sehen ja auch z. B. an der Irismuskulatur in der Regel, wenn sie an den Krampfzuständen der Tetanie sich beteiligt, eine Miosis, also eine Oculomotoriuswirkung zustande kommen. Wie unser Fall 4 aber beweist, kann auch gelegentlich eine Mydriasis, also eine Sympathicuswirkung beobachtet werden. - Meine drei Fälle sind im übrigen keineswegs genügend, um irgend etwas in dieser Frage zu beweisen. Vielleicht geben sie aber Veranlassung, diesen Verbältnissen bei der Sektion einschlägiger Fälle genauere Beachtung zu schenken. Wichtig wäre dann freilich auch, die Sektion möglichst rasch nach erfolgtem Tode auszuführen.

Es sei an dieser Stelle noch kurz auf die klinischen Erscheinungen verwiesen, die in der Krankengeschichte des Falles 4 notiert sind, bei dem ich den Herztod selbst miterlebte. Hier sank die Frequenz der Herzschläge rapid ab, die Kontraktionen wurden immer langsamer und hatten nach 1-2 Minuten ganz aufgehört. Ob sich der Verlauf immer in dieser Weise abspielt, können nur weitere Beobachtungen lehren. Der Arzt hat nur selten Gelegenheit dazu, besonders da sein ganzes Handeln, wenn ibn der Zufall zum Zeugen einer solchen Katastrophe macht, von therapeutischen Gesichtspunkten beherrscht sein muss.

Wenn wir der speziellen Ätiologie des Herztodes im Verlauf der Säuglingstetanie nachforschen, uns dịe Momente vergegenwärtigen, die beim Tetaniker als Anlässe zur Synkope bekannt sind, so ergeben sich einige Gesichtspunkte, die sich zu gunsten meiner Auffassung geltend machen lassen.

Als solche Anlässe sind hier vor allem schwere Stimmritzenoder sonstige Respirationskrämpfe zu nennen; ferner der Status lymphaticus bezw. die Hyperplasie der Thymusdrüse, die doch in einer beträchtlichen Zahl derartiger Fälle bei der Sektion gefunden wird. Wir wissen ferner - die Tatsache ist jüngst von $\mathrm{Feer}^{1}$ ) wieder besonders betont worden -, dass tödlicher Stimmritzenkrampf sich auffallend gern an grosse Mahlzeiten anschliesst. In meinem Fall 4 erfolgte der plötzliche Herztod (ohne Stimmritzenkrampf) wenige Minuten nach der Schlundsondenfütterung. Ob die Bauchlage im Fall 6 als auslösendes Moment mitgewirkt hat, ist schwer zu entscheiden; die Möglichkeit muss jedenfalls in Betracht gezogen werden.

All die genannten Momente sind geeignet, auf das Herz oder seine Nerven mechanische oder zirkulationsbehindernde Wirkungen

1) E. Feer, l. c. S. 726. 
auszuüben. Wir wissen, dass diese Momente auch in der Pathogenese der manifesten Tetanie eine Rolle spielen können. Ich erinnere an das Trousseausche Phänomen, bei dessen Zustandekommen wahrscheinlich beide Ursachen zusammenwirken. Man könnte versucht sein, Analogien für die Herztetanie zu konstruieren. Bei der Magenfüllung als Ursache des Tetanietodes ist wohl auch die direkte mechanische Wirkung auf das Herz und seine Nerven sehr ins Auge zu fassen.

Immerhin gibt es aber auch eine andere Erklärungsmöglichkeit, die mir die wahrscheinlichere dünkt. Der Magen wird ebenso wie das Herz vom Vagus und vom Sympathicus innerviert. Es wäre daher ein nervöser reflektorischer Zusammenhang mit den Innervationsstörungen des Herzens leicht zu begreifen. ${ }^{1}$ ) Noch leichter wäre das für die Asystolie im Anschluss an den Glottis- oder Respirationskrampf; die Nervenbahnen, die dieses Muskelgebiet beherrschen, verlaufen auch im Vagus. Wir wissen, dass häufig im Anschluss an den Laryngospasmus allgemeine Konvulsionen auftreten, dass also eine Irradiation auf andere Muskelgebiete stattfindet; es wäre ein analoges Übergreifen des Reizes auf die Herznerven, die im Vagus verlaufen (bezw. auf deren Zentren), sehr gut denkbar. Man könnte sich andererseits auch vorstellen, dass die gleichen Einflüsse, die den Stimmritzenkrampf auslösen - und hier wären nach unseren Erfahrungen auch psychische Momente, Schreck, Aufregung, heftiges Schreien usw. besonders zu berücksichtigen - nicht nur spasmogen auf die Atemmuskeln, sondern in gleicher Weise auch auf das Herz selbst, bezw. auf die Zentren, die die Herzbewegung regulieren, einwirken. Obige Annahme würde erheblich an Wahrscheinlichkeit gewinnen, wenn es etwa gelänge, gelegentlich während des Glottiskrampfes eine auffallende Frequenzänderung der Herzkontraktionen, also etwa eine Bradykardie zu konstatieren. Ich habe vorerst keine eigenen Beobachtungen in dieser Richtung anstellen können.

Beim Studium dieser Fragen drängt sich das Bedürfnis auf, Untersuchungen anzustellen, um diese mehr hypothetischen klinischen Erwägungen auf ihre Stichhaltigkeit zu prüfen. Wenn ich auch glaube, dass ein genaues klinisches Studium einschlägiger Fälle noch manche Aufklärung bringen wird, so scheinen mir doch auch experimentelle Fragestellungen recht wohl möglich. Durch Exstirpation der Epithelkörperchen ist man ja in der Lage, eine der menschlichen Tetanie ähn-

1) Auf die Finkelsteinsche Beobachtung von Tachypnoe und Tachykardie im Anschluss an Magenspülung sei auch in diesem Zusammenhang noch ein Mal hingewiesen. 
liche Krankheit bei Hunden, Ratten usw. zu erzeugen. Vielleicht lässt sich an solchen Tieren auch der Nachweis einer gesteigerten Erregbarkeit der Herznerven führen. - Freilich haben wir noch nicht die Berechtigung, die parathyreoprive Erkrankung der Tiere ohne weiteres mit der menschlichen Tetanie zu identifizieren, am wenigsten mit der Säuglingstetanie, die sich ja in wesentlichen Punkten - und nicht zum mindesten durch die Gefahr des plötzlichen Herztodes - von der Tetanie der Erwachsenen unterscheidet.

Auch an eine direkte Prüfung der Herzerregbarkeit bei tetaniekranken Säuglingen könnte man denken. Bei Erwachsenen wurde etwas $\ddot{A}$ hnliches schon einmal versucht. In Analogie mit Ziemssens Untersuchungen an dem freiliegenden Herz der K. S., das durch stärkere elektrische Ströme zu rascherer Schlagfolge gebracht werden konnte, untersuchte Franz Müller ${ }^{1}$ ) den Einfluss solcher durch die Thoraxwand auf das Herz übertragener stärkerer galvanischer Ströme. Es gelang ihm aber nicht, bei Tetaniekranken einen deutlichen Einfluss auf die Pulsfrequenz nachzuweisen. Für die von mir angeregte Frage wäre allerdings eher der Versuch einer Reizung des Nervus vagus, etwa am Halse von Wichtigkeit. Müller gelangte auch hier bei Erwachsenen zu keinem Resultat. Doch ist nicht zu vergessen, dass bei Erwachsenen der Herztod ebenso wie der Glottiskrampf, keine Rolle im Krankheitsbild der Tetanie spielt. Bei tetaniekranken Säuglingen möchte ich aber solche Versuche für recht gewagt halten, da wir gar nicht wissen können, ob sie nicht dadurch gefährdet sind.

Die Beachtung der glatten Muskulatur und des Herzens, des Sympathicus und des vegetativen Systems bei der Säuglingstetanie, zu der ich hoffe durch obige Mitteilungen angeregt $\mathrm{zu}$ haben, ist vielleicht nicht nur klinisch von Interesse.

Man ist heute vielfach geneigt, den Epithelkörpern in der Pathogenese der Tetanie eine besonders wichtige Rolle zuzuerkennen. Die Forschung bat sich in dieser Richtnng lediglich auf die Feststellung pathologischer Veränderungen in den Epithelkörpern verstorbener Kinder beschränkt.

Wenn auch die Untersuchungen von $\operatorname{Erdheim}^{2}$ ) und Yanase $^{3}$ )

1) Franz Müller, Über das elektrische Verhalten des Herzens bei Tetanie. Deutsches Arch. f. klin. Med. 1898. Bd. 61. S. 632.

2) J. Erdheim, Tetanie parathyreopriva. Mitteilungen aus den Grenzgebieten der inneren Medizin und Chirurgie 1906. Bd. 16. S. 632 und Mitteilungen der Gesellschaft für innere Medizin und Kinderheilkunde. Wien, 22. XI. 1906.

3) J. Yanase, Verhandlungen der Gesellschaft für Kinderheilkunde in 
hier viel Positives zutage förderten, so haben sie doch wohl nicht vermocht, für die Epithelkörpertheorie unumstössliche Beweise beizubringen. Auch sind die Untersuchungen anderer Forscher z. T. nicht im gleichen Sinne ausgefallen. So berichten Schiffer und Rheindorf ${ }^{1}$ ), dass sich in einem schweren, tödlich endenden Tetaniefall bei einem 4 Jahre alten Kinde - es scheint auch eine typische Herztetanie gewesen zu sein - keine Veränderungen an den Epithelkörpern auffinden liessen (Untersuchung sämtlicher vier Epithelkörper in Serienschnitten). Auch in meinen Fällen wurde nichts gefunden; allerdings wurden nicht sämtliche Epithelkörper in Serienschnitte zerlegt. Esc he$\mathrm{rich}^{2}$ ) betont die Möglichkeit rein funktioneller Beeinträchtigung bezw. Minderwertigkeit dieser Organe. Es müsste dann aber auch möglich sein, im Leben Stoffwechselstörungen aufzudecken, die auf eine Schädigung dieser Drüsen schliessen lassen. Bisher ist hauptsächlich der Kalkstoffwechsel untersucht worden. Es hat sich hier ein Resultat ergeben, das mit der Epithelkörperchentheorie recht gut übereinstimmt. Nach den Untersuchungen von $\mathrm{Cybulski}^{3}$ ) und $\mathrm{Schabad}^{4}$ ) ist bei tetaniekranken Kindern der Kalkstoffwechsel gestört; sie zeigen ein mangelhaftes Kalkretentionsvermögen bezw. gesteigerte Kalkabgabe. Eine ganz analoge Störung des Kalkstoffwechsels haben Mc Callum und Vögtlin ${ }^{5}$ ) an parathyreopriven Huiden nachgewiesen.

Durch die Arbeiten der Wiener Schule durch Eppinger, Falta, Rudinger ${ }^{5}$ ) wissen wir, dass zwischen den Epithelkörpern und den anderen Organen mit innerer Sekretion Beziehungen bestehen, dass z. B. die Epithelkörper speziell einen hemmenden Einfluss auf das chromaffine System und den Sympathicus ausüben sollen. Bekannt ist andererseits die geringe Ausbildung des chromaffinen Systems bei

Dresden 1907. S. 11 u. Jahrbuch f. Kinderheilkunde. 1908. Bd. 67. Ergänzungsheft S. 57 .

1) Schiffer u. Rheindorf, Familiäre Tetanie. Verein f. inn. Med. u. Kinderheilkde. Berlin, 30. V. 10. - Ref. Deutsche mediz. Wochenschr. 1910. S. 1297.

2) Th. Escherich, Zur Kenntnis der tetanoiden Zustände des Kindes. alters. Verhandlungen der Gesellschaft für Kinderheilkunde in Dresden 1907. S. 22 .

3) Th. v. Cybulski, Über den Kalkstoffwechsel des tetaniekranken Säuglings. Monatsschr. f. Kinderheilkde. 1906. Bd. 5. S. 409.

4) J. A. Schabad, Der Kalkstoffwechsel bei Tetanie. Monatsschrift f. Kinderheilkde. 1910. Bd. 9. S. 25.

5) Me Callum u. Vögtlin, Journ. of experim. Medicine 1909. Nr. 1.

6) H. Eppinger, W. Falta u. C. Rudinger, Über die Wechselwirkung der Drüsen mit innerer Sekretion. 2. Mitteilung. Zeitschr. f. klin. Med. 1909. Bd. 67. S. 380 . 
Lymphatikern (Wiesel, Hedinger), die wiederum durch die Tetanie besonders gefährdet sind, wie oben hervorgehoben wurde. Man sieht, es wäre leicht, Hypothesen zu bauen; das ist keineswegs meine Absicht; ich glaube aber doch, dass die Ausblicke, die sich auf diesem Gebiete eröffnen, daza auffordern, beim Sammeln klinischer Beobachtungstatsachen auch das vegetative System und den Sympathicus zu berücksiehtigen. Wenn einmal später die physiologischen Erkenntnisse in diesen schwierigen Problemen auf gesicherten Grundlagen ruhen, kann man hoffen, dass auch die Beobachtungen pathologischer Vorgänge sich zu einem festen Gebäude zusammenfügen werden. 